\title{
Alzheimer’s Disease Drug Discovery may be Misled by Wrong Animal Models
}

\section{Amir Nazem*}

Center for Neurosciences and Elmezzi Graduate School of Molecular Medicine, Feinstein Institute for Medical Research, NY, USA

*Corresponding author: Amir Nazem, Center for Neurosciences and Elmezzi Graduate School of Molecular Medicine, Feinstein Institute for Medical Research, Manhasset, NY 11030, USA, Tel: 5165621333; E-mail: anazem@nshs.edu

Rec date: June 26, 2014; Acc date: June 28, 2014; Pub date: June 30, 2014

Copyright: (C) 2014 Nazem A. This is an open-access article distributed under the terms of the Creative Commons Attribution License, which permits unrestricted use, distribution, and reproduction in any medium, provided the original author and source are credited.

\section{Editorial}

Alzheimer's disease $(\mathrm{AD})$ is one of the most expensive human diseases and a leading cause of disability and death among elderly. As the elderly population is growing worldwide, $\mathrm{AD}$ will soon become a major human healthcare challenge if it remains incurable [1]. The currently available therapeutics for $\mathrm{AD}$ only acts to lower its symptoms without any effect on the actual underlying pathology [2].

In recent years, however, significant amount of research has been focused on finding the so called "neuroprotective" agents, therapeutics that can stop or slow the disease progress by targeting special molecular mechanisms in the $\mathrm{AD}$ pathology process. Yet more futuristic are approaches that can rebuild the damaged tissue, called as "regenerative agents". These two approaches together are known as "disease-modifying approaches" [3]. According to its definition, a disease modifying treatment should not only interfere with the underlying pathological mechanisms leading to neuronal dysfunction and loss in $\mathrm{AD}$, but it should also lead to significant clinical improvement of the patient [3].

In the past two decades, several hundreds of potential disease modifying agents has shown to be effective in animal models of AD, but all have failed in the phase three clinical trials [4]. These failures question both the basis on which the therapeutic agents have been designed and the animal models used in the pre-clinical phases [5]. The most commonly used animal models of $\mathrm{AD}$ are based on the lesions of $\mathrm{AD}$, Amyloid- $\beta(\mathrm{A} \beta)$ plaques and neurofibrillary tangles. These models are transgenic carriers of mutations related to amyloid or tau production (amyloid precursor protein (APP), presenilin-1 (PS1) and PS2 or tau mutations). However, the mentioned mutations do not cause late-onset $\mathrm{AD}$ (LOAD).

Admittedly, our today's in-depth understanding of potential mechanisms involved in LOAD pathology is considerably based on studies performed on the conventional transgenic models of $\mathrm{AD}$ [5]. However, these $\mathrm{AD}$ animal models are designed to recapitulate $\mathrm{AD}$ lesions through an etiology similar to rare familial types of AD. Since LOAD is not caused by such mutations, the findings from these animal models cannot be reliably translated to human LOAD.

In contrast to rare genetic forms of $\mathrm{AD}$, the molecular triggers and pathways leading to LOAD is still unclear and highly controversial [6]. Studies that point to the more primary stages in AD pathogenesis have provided evidence of different yet overlapping molecular triggers of LOAD, emerging even before $A \beta$ and tau pathologies. Those molecular mechanisms include mitochondrial dysfunction, oxidative stress, hypoxia, chronic neuroinflammation, trace metal dyshomeostasis, cell cycle reentry, vascular pathology and insulin resistance $[1,7]$. Currently, however, these pathological paradigms of LOAD lack wellestablished in vivo models to be used in pre-clinical drug discovery studies [5,6]. Such etiology-based models of LOAD may provide breakthroughs in future AD drug discovery.

\section{References:}

1. Nazem A, Mansoori GA (2011) Nanotechnology for Alzheimer's disease detection and treatment. Insciences J 1: 169-193.

2. Nazem A, Mansoori GA (2014) Nanotechnology Building Blocks for Intervention with Alzheimer's Disease Pathology: Implications in Disease Modifying Strategies. J Bioanal Biomed 6: 009-014.

3. Cummings JL (2009) Defining and labeling disease-modifying treatments for Alzheimer's disease. Alzheimers Dement 5: 406-418.

4. Mangialasche F, Solomon A, Winblad B, Mecocci P, Kivipelto M (2010) Alzheimer's disease: clinical trials and drug development. Lancet Neurol. 9: 702-716.

5. Sabbagh JJ, Kinney JW, Cummings JL (2013) Animal systems in the development of treatments for Alzheimer's disease: challenges, methods, and implications. Neurobiol Aging 34: 169-183.

6. Castellani RJ, Perry G (2012) Pathogenesis and disease-modifying therapy in Alzheimer's disease: the flat line of progress. Arch Med Res 43: 694-698.

7. Nazem A, Mansoori GA (2008) Nanotechnology solutions for Alzheimer's disease: advances in research tools, diagnostic methods and therapeutic agents. J Alzheimers Dis 13: 199-223. 